\title{
A Study on the Characteristics Influencing the Pressure at the Root of a Distributed One-Dimensional Model of Arterial Blood Flow
}

\author{
Shima Abdullateef ${ }^{1}$, Jorge Mariscal-Harana ${ }^{2}$, Jordi Alastruey ${ }^{2}$, Ashraf W Khir ${ }^{1}$ \\ ${ }^{1}$ College of Engineering, Design, and Physical Sciences, Brunel University London, Middlesex, UK \\ ${ }^{2}$ School of Biomedical Engineering and Imaging Sciences, King's College London, London, UK
}

\begin{abstract}
Blood pressure carries crucial information about the response of the arterial system to the beating heart. Extracting useful information from the blood pressure plays a significant role in the diagnosis and treatment of cardiovascular disease such as hypertension.

There are many studies focusing on the existence of reflection waves in the ascending aorta and their influence on the amplitude of pressure. However, there is an ongoing debate about their origin, and the distance that a reflection wave can travel.

In this study, a one-dimensional (1D) model of a series of bifurcations was used to analyse the effect of bifurcations on the pressure amplitude. A comparison was made between the pressure in the inlet of the model and in the terminal ends. Results showed an exponential decay with increasing numbers of bifurcations with no reflections reaching the inlet after the 7th generation of bifurcations. Therefore, a single reflection originating at the periphery may not be discernible at the aortic root.
\end{abstract}

\section{Introduction}

Blood pressure waves generated by heart contraction encounter many bifurcations in the arterial tree, generating backward waves travelling towards the heart [1]. The existence of backwards waves, their magnitude, and their effect on increasing the pressure measured in the aortic root is an ongoing debate, particularly for the outstanding question about the origin of the reflected wave present in the aortic root, and how far the reflected waves may travel in the arterial tree [2].

Haemodynamic simulations using computational models can be used to assess different vessels in the systemic circulation that might not be accessible in humans, or in certain conditions cannot be achieved in vivo. 1D modelling has the ability to capture the global dynamics of the arterial system, and it has been validated against in vitro [3] and in vivo experiments $[4,5]$. The aim of this study is to investigate how bifurcations affect the magnitude of reflected waves, and how far reflected waves can travel towards the heart with a discernible magnitude. We illustrate this by considering a series of bifurcations ended by outflow boundary conditions that completely absorb any incoming wave in order to extract the reflections originating from the bifurcations only.

\section{Methods}

The non-linear, 1D equations of blood flow in elastic tubes have been used to simulate blood flow. The equations consider blood as an incompressible and Newtonian fluid. This model is used to trace the waves as they travel through a branching system to mimic the arterial system. For each segment in the arterial system, the physical principles of conservation of mass and momentum is applied and they can be written respectively as:

$$
\begin{gathered}
\frac{\partial A}{\partial t}+\frac{\partial(A U)}{\partial x}=0 \\
\frac{\partial U}{\partial t}+U \frac{\partial U}{\partial x}=\frac{-1}{\rho} \frac{\partial P}{\partial x}+\frac{f}{\rho A}
\end{gathered}
$$

where $x$ is the axial coordinate along the segments, $t$ is time, $U(x, t)$ is the mean blood axial velocity, $A(x, t)$ is the cross-sectional area of each segment, $P(x, t)$ is the internal pressure in each cross-section, and $\rho=$ $\left.1050 \mathrm{Kg} \mathrm{m}^{-3}\right)$ is the density of the blood. The friction force per unit length is given by $f=-2(\gamma+2) \mu \pi U$, where $\gamma$ is determined by the prescribed shape of the velocity profile. Based on findings of [6], $\gamma=9$ is in a good agreement with experimental data and considers the velocity profile to be flat with no-slip condition. The viscosity of blood, $(\mu)$, is $4 \mathrm{mPa}$ s. Equations (1) and (2) contain three unknown variables namely pressure, velocity, and area. Therefore, the following pressure-area relationship is coupled to the governing equations to close the system of equations,

$$
P=P_{0}+\frac{\beta}{A_{0}}\left(\sqrt{A}-\sqrt{A_{0}}\right) .
$$


Where $\beta$ is

$$
\beta=\frac{4}{3} \sqrt{\pi} h E
$$

with $A_{0}(x)$ as the area in initial condition where $(P, U)=$ $\left(P_{0}, 0\right), h(x)$ as wall thickness, and $E(x)$ as Young's modulus. This law assumes the arterial wall to be thin, elastic, homogeneous, and incompressible. The resulting pulse wave velocity, $c(x)$, was calculated using the MoensKorteweg equation:

$$
c=\sqrt{\frac{\beta}{2 \rho A_{0}}} A^{\frac{1}{4}} .
$$

Injecting a perturbation into a vessel induces a wave that propagates with speed of $U+c$ in the forward direction and $U-c$ in the backward direction. The resulting changes in pressure and velocity in the arterial tree can be separated into the forward-travelling and the backward-travelling components, where the forward direction is away from the heart to the periphery and the backward direction is from the periphery towards the heart. The changes in total pressure and velocity are the sum of changes in their forward and backward components, $d P=d P_{f}+d P_{b}$ and $d U=d U_{f}+d U_{b}$. By using these equations and the waterhammer equation,

$$
d P_{f}=\rho c d U_{f}, \quad d P_{b}=-\rho c d U_{b}
$$

the forward and backward components of pressure and velocity are:

$$
d P_{f, b}=\frac{1}{2}(d P \pm \rho c d U), \quad d U_{f, b}=\frac{1}{2}\left(d U \pm \frac{d P}{\rho c}\right) .
$$

Wave intensity analysis (WIA) studies the flux of energy carried by the wave per cross-sectional area, $d I$. A positive value for WIA $(d I>0)$ shows forward travelling waves, and a negative value $(d I<0)$ shows their reflections. WIA is the product of changes of pressure and velocity during a small time interval. $d I$ has SI units of $W / m^{2}$ and provides an insight if the direction and timing of the waves. Using Equations (6) and (7), $d I$ can be calculated by:

$$
d I_{f, b}=d P_{f, b} d U_{f, b}=\frac{ \pm 1}{4 \rho c}(d P \pm \rho c d U)^{2} .
$$

Since the length of the segments $(l)$ is fixed and $c$ can be calculated with predefined material properties, the transient time is given by:

$$
t=\frac{l}{c}
$$

Many studies have shown evidence that arterial bifurcations are well-matched for forward-travelling waves, and poorly-matched for backward-travelling waves. Based on the measurements of human bifurcations in different sites of an arterial system, area ratio between the daughters and the parent vessels of $1.18 \pm 0.04$ was obtained [7]. Therefore, in this study the relation between the parent and the daughter segments is $\alpha=1.15$, where

$$
\alpha=\frac{\left(A_{D 1}+A_{D 2}\right)}{A_{M}} .
$$

$A_{M}, A_{D 1}$, and $A_{D 2}$ are the cross-sectional areas of the mother tube, 1 st daughter tube, and 2 nd daughter tube respectively. With $\alpha=1.15$, the forward wave is travelling the bifurcation, with no reflection waves created from the bifurcation. For a detailed explanation of the numerical modelling we refer the reader to [8]. On the basis of using the above-mentioned equations, two sets of 1D simulations were carried out. The length of all the segments are 5 metres in order to prevent the overlapping of wave reflections.

\subsection{5 consecutive bifurcations}

In the first experiment, a Gaussian-shaped pulse was inserted from the inlet of the mother tube followed by 5 generations of bifurcations (Figure 2.1). With this model, the changes in pressure in the inlet are monitored while there is a total blockage in the 5 th bifurcation. The boundary

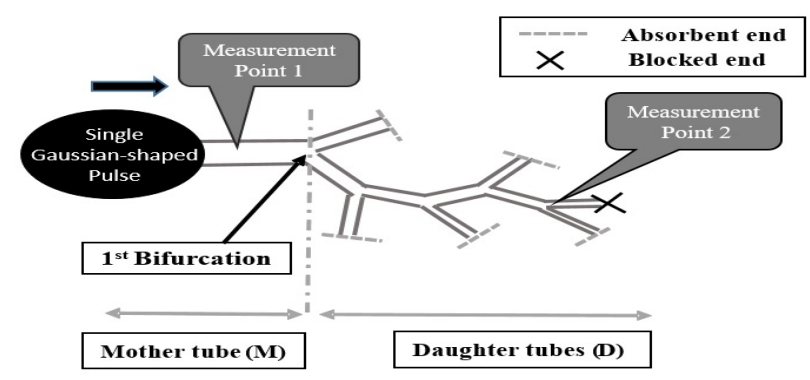

Figure 1. Schematic representation of the 5 consecutive bifurcation structure with the Gaussian-shaped pulse inserted in the inlet. Each segment is 5 metres long.

conditions for the other terminal branches are completely absorbent.

\subsection{5 consecutive bifurcations}

A structure of 15 consecutive bifurcations was simulated using a distributed 1D model. The changes in pressure in the inlet of the mother tube (M) were analysed in 15 different simulations (Figure 2.2). In each simulation, a Gaussian-shaped pulse is inserted in the terminal point of the daughter tubes one at a time. The pressure calculated in the inlet of the mother tube was compared with the amplitude of input pressure from the peripheral points. The 


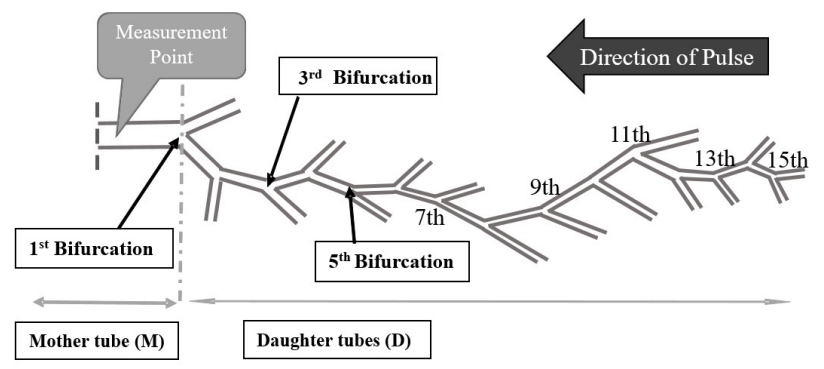

Figure 2. Schematic representation of the 15 consecutive bifurcation structure.

boundary condition of the other terminals were kept absorbent, in order to investigate the magnitude of the arrival waves from the specific terminal point to the mother tube.

\section{Results}

\subsection{5 consecutive bifurcations}

Since the length and the pulse wave velocity of the segments are known, the transient time of the reflections can be calculated theoretically using Equation (9). Pressure and velocity variations in measurement point 1 is shown in Figure 3. The reflection originating from the blocked terminal end in the 5th bifurcation arrived at the inlet of the mother tube at 7.98 seconds. At 7.98 seconds the existing amplitude of pressure is negligible compared to the input and overlapped with other artefacts whose source cannot be identified.

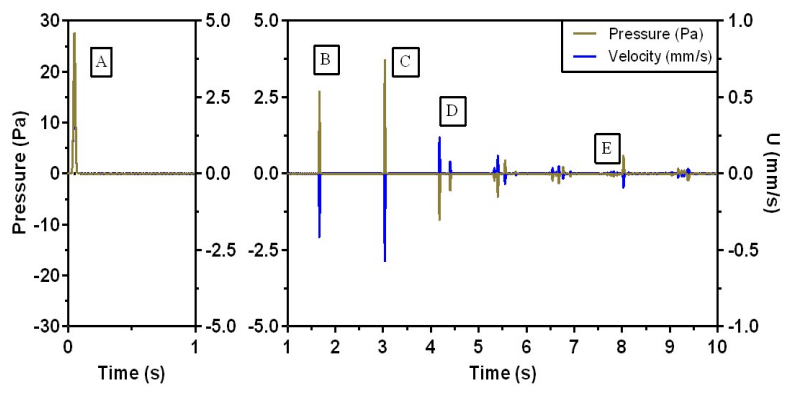

Figure 3. Pressure and velocity variations in the mother tube of the 5 bifurcations. Each label in the figure corresponds to a wave. The left-hand figure is the input wave and the right-hand side is a magnified view of the rest of pressures and velocities.

WIA is used for the measurements in the inlet of the structure and in the blocked segment (Figures 4 and 5). There is no evidence of the reflection from the blocked end in the mother tube.
Table 1. Arrival times and corresponding pressures in the inlet of the mother tube, as shown in Figure 3.

\begin{tabular}{|c|c|c|c|}
\hline \multicolumn{4}{|c|}{ Measurement Point 1 } \\
\hline Wave & Path & Arrival Time (s) & Pressure (Pa) \\
\hline A & Input & 0.00 & 28.00 \\
\hline B & $1,-1$ & 1.67 & 2.76 \\
\hline C & $1,2,-2,-1$ & 3.02 & 3.74 \\
\hline D & $1,2,3,-1,-2,-3$ & 4.18 & -1.54 \\
\hline E & $1,2,3,4,5,-5,-4,-3,-2,-1$ & 7.98 & 0.65 \\
\hline
\end{tabular}

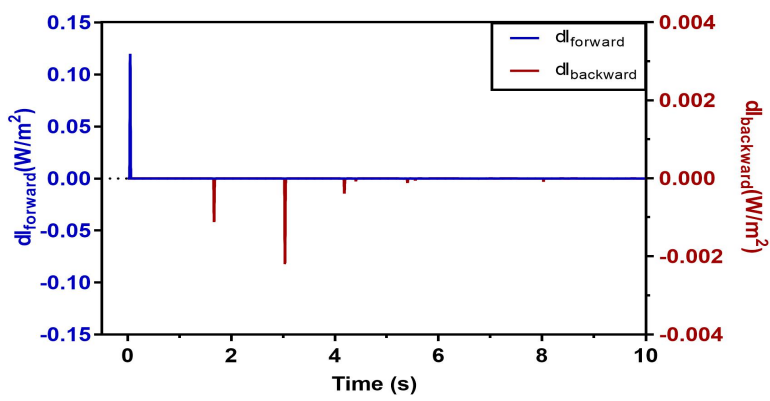

Figure 4. WIA in the first measurement point. The effect of reflection from the first and second bifurcations can be seen at $1.6 \mathrm{~s}$ and $3 \mathrm{~s}$, respectively.

\subsection{5 consecutive bifurcations}

In these sets of simulations we imposed an 'artificial reflection' large enough to be detected in the inlet of the mother tube. The pressure measured in the mother tube, $\mathrm{P}(\mathrm{M})$, was compared against the pressure that was imposed in terminal points of each daughter tubes, $\mathrm{P}(\mathrm{D})$, and the results are presented as a ratio ( $\mathrm{Rp})$ of the measured pressures (Figure 3.2). The results show a significant decay in the amplitude of reflection waves reaching the root of the mother tube, which may indicate that more than half of each wave's amplitude was re-reflected and trapped between each bifurcation.

\section{Discussion}

Similar to many branches of applied mathematics, it is necessary to analyse simple models before increasing the complexity of the modifying feature present in reality. Therefore, we started by considering a structure of segments without peripheral reflections and 5-metre segments. Compared to the existing models of the arterial system in the literature, the models studied here are relatively simple. However, they reproduce the features of reflected waves in a branching system similar to the arterial tree by easy-to-grasp mechanisms. The absence of reflected waves generated from other terminal points might be an oversim- 


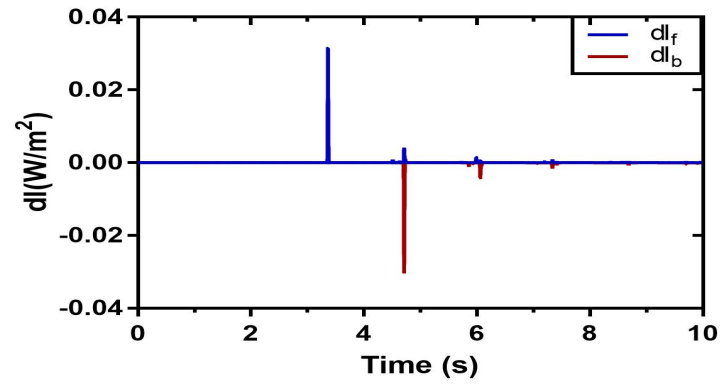

Figure 5. WIA in the measurement point 2 in the blocked segment.

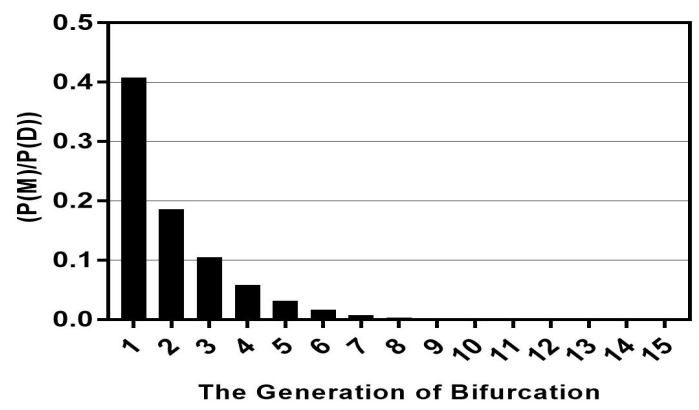

Figure 6. Ratio of the measured pressure in the mother tube, $\mathrm{P}(\mathrm{M})$, over the pressure measured in the daughter tubes, $\mathrm{P}(\mathrm{D})$, in 15 generation of bifurcations.

plification, but it allowed the tracking of changes in pressure waveform passing different bifurcations. In addition, although not physiological, the length of 5 metres of each segment was used in order to prevent the overlapping of waves. In contrast to the arterial system, with multiple sites of reflection from tapering and peripheral resistances, the models in this study focus on the effect of the reflections solely because of bifurcations.

Based on the results of the first experiment, reflected waveforms are discernible when originating from the two initial bifurcations. As the wave travels throughout the model, the magnitude of the reflection decreases and even the reflection from the blocked terminal point is too small to be discerned without magnification of the time period 10-15s. In the second part of this study, the reflection waves from the terminal points were considerable enough to be observed in the inlet of the structure even after travelling through 15 reflection sites. The results of these simulations provided an overview of the ratio of reflections originating from the end point of different generation of bifurcations. This may indicate that more than half of the waves are re-reflected in the arterial tree.

\section{Conclusion}

A significant decrease in the amplitude of the reflected wave reaching the inlet of the mother segment is evident in both experiments. With the increase in number of bifurcations, as is the case in vivo, single reflections originating at the periphery may not be discernible at the aortic root. Further work is required to examine the decay of the reflected waves in a larger number of segments and bifurcations similar to those of the arterial system. Introducing the effect of tapering vessel can give more insight about the propagation of pressure in the arterial system.

\section{Acknowledgements}

This study was funded by a Brunel University London PGR scholarship.

\section{References}

[1] Khir AW, Parker KH. Measurements of wave speed and reflected waves in elastic tubes and bifurcations. J Biomech 2002;35(6):775 - 783.

[2] Segers P, O'Rourke MF, Parker KE, Westerhof N, Hughes AD. Towards a consensus on the understanding and analysis of the pulse waveform: Results from the 2016 workshop on arterial hemodynamics: Past, present and future. Artery Res 2017;18:75-80.

[3] Alastruey J, Khir AW, Matthys KS, Segers P, Sherwin SJ, Verdonck PR, Parker KH, Peiró J. Pulse wave propagation in a model human arterial network: Assessment of 1 dimensional visco-elastic simulations against in vitro measurements. J Biomechanics 2011;44(12):2250 - 2258.

[4] Willemet M, Lacroix V, Marchandise E. Inlet boundary conditions for blood flow simulations in truncated arterial networks. J Biomechanics 2011;44(5):897 - 903.

[5] Li Y, Gu H, Fok H, Alastruey J, Chowienczyk P. Forward and backward pressure waveform morphology in hypertension. Hypertension 2017;69(2):375-381.

[6] Smith NP, Pullan AJ, Hunter PJ. An anatomically based model of transient coronary blood flow in the heart. SIAM J Appl Math 2001;62:990-1018.

[7] Papageorgiou GL, Jones BN, Redding VJ, Hudson N. The area ratio of normal arterial junctions and its implications in pulse wave reflections. Cardiov Res 1990;24(6):478-484.

[8] Alastruey J, Parker KH, Peiró J, Sherwin SJ. Analysing the pattern of pulse waves in arterial networks: a time-domain study. J Eng Math 2009;64(4):331-351.

Address for correspondence:

Professor Ashraf W Khir

Brunel University London, Kingston Lane, Uxbridge, Middlesex, UB8 3PH

Ashraf.Khir@brunel.ac.uk 